\section{Surgical management of nasal disease}

\section{Samantha Woods}

Nasal disease in dogs and cats is common, with many patients presenting for investigation of nasal discharge. With the ability now for many diseases to be more thoroughly investigated using advanced imaging, and treated using rhinoscopy and more minimally invasive methods, surgery has become less important overall in the management of nasal disease. There are still, however, some conditions requiring surgical intervention including treatment of stenotic nares (brachycephalic breeds), removal of tumours of the nasal planum, management of fungal infection, trauma and some foreign bodies and biopsy of lesions within the nasal cavity or nasopharynx.

The most common tumour of the nasal planum in dogs and cats is squamous cell carcinoma (SCC), although others do occur. Nasal planum SCC tends to be more locally invasive into soft tissues and bone than metastatic to distant sites, so surgical resection with or without adjunctive radiation therapy can be curative. Excision often requires large unilateral or more radical bilateral excisions of the nasal planum to achieve appropriate margins.

Carcinomas are the most commonly identified malignant tumours of the canine nasal cavity. Once again, these are slow growing and locally invasive rather than distantly metastatic, however surgery alone to remove these does not increase good survival rates. Treatment of choice is radiation therapy, with or without cytoreductive surgery. Biopsy for diagnosis is often the only surgery necessary in these cases and can be performed through a dorsal rhinotomy or a ventral palate approach depending on the location of the tumour.

Aspergillus fumigatus infection is the most common cause of fungal rhinosinusitis in dogs. The infection causes plaque-like lesions within the airspaces of the nasal cavity that are resistant to systemic antifungals. Therapy usually involves endoscopic debridement and non-invasive placement of infusion catheters into the nares to allow instillation of antifungals. Surgical trephination of the sinuses and placement of catheters invasively may be required for treatment of more resistant cases.

Stenotic nares are a common presentation of brachycephalic syndrome in dogs although some cats are also affected. The deviation of the dorsolateral wing cartilage in these breeds leads to significant airway obstruction and associated secondary effects. Different techniques are described including the basic 'Trader's technique', horizontal or vertical wedge resection and combination methods of wedge resection/alarpexy or ala-vestibuloplasty, all aimed at improving air flow through the nostril and reducing turbulence at the level of the larynx.

Rhinotomy is indicated in patients with benign mass lesions, chronic and undiagnosed nasal discharge, challenging fungal rhinitis cases, patients in which nasal tooth displacement occurs and some nasal tumours whether for treatment or biopsy.

This session aims to discuss the surgical procedures mentioned above, with case-based examples of nasal planum resection and reconstruction to highlight principles of nasal oncological surgery. The different techniques for management of stenotic nares will also be discussed.

\section{KEY LEARNING OBJECTIVES}

- Whilst many nasal diseases can be managed medically, surgery is still indicated in certain conditions

- Surgery is the mainstay of treatment of nasal planum neoplasia, with the potential to provide 'cure' in some cases

- Brachycephalic patients benefit from early correction of stenotic nares

\section{MULTIPLE CHOICE QUESTIONS}

1. What is the most common nasal planum tumour seen in cats and dogs?
(A) Adenocarcinoma
(B) Lymphoma
(C) Mast cell tumour
(D) Squamous cell carcinoma

2. What are the 'primary' abnormalities detected in brachycephalic dogs, that if corrected early may slow progression of other components of the syndrome?
(A) Elongated soft palate and aberrant nasal turbinates
(B) Elongated soft palate and stenotic nares
(C) Stenotic nares and aberrant nasal turbinates
(D) Stenotic nares and tonsillar hypertrophy

3. You are performing investigations in a 10-year-old Border Collie with a history of chronic unilateral haemorrhagic nasal discharge. Radiographs identify lysis of the hard palate and erosion of the nasal septum and you suspect a tumour. What is the most appropriate surgical approach to confirm your diagnosis?

(A) Dorsal rhinotomy

(B) Lateral rhinotomy

(C) Rostral rhinotomy

(D) Ventral rhinotomy 